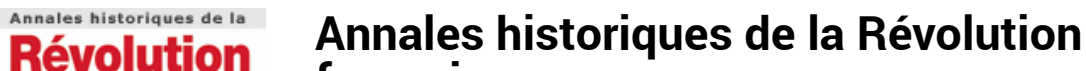

française française

328 | avril-juin 2002

La Révolution et le Droit

\section{Gaspard Monge. Le fondateur de Polytechnique}

\section{Patrice Bret}

\section{(2) OpenEdition}

\section{Journals}

Édition électronique

URL : https://journals.openedition.org/ahrf/2261

DOI : 10.4000/ahrf.2261

ISSN : 1952-403X

\section{Éditeur :}

Armand Colin, Société des études robespierristes

\section{Édition imprimée}

Date de publication : 1 juin 2002

Pagination : 233-235

ISSN : 0003-4436

\section{Référence électronique}

Patrice Bret, "Gaspard Monge. Le fondateur de Polytechnique », Annales historiques de la Révolution française [En ligne], 328 | avril-juin 2002, mis en ligne le 11 mai 2006, consulté le 23 avril 2022. URL : http://journals.openedition.org/ahrf/2261 ; DOI : https://doi.org/10.4000/ahrf.2261

Ce document a été généré automatiquement le 23 avril 2022.

Tous droits réservés 


\title{
Gaspard Monge. Le fondateur de Polytechnique
}

\author{
Patrice Bret
}

\section{RÉFÉRENCE}

François Pairault, Gaspard Monge. Le fondateur de Polytechnique, Paris, Tallandier (Coll.

Biographies - Figures de proue), 2000, 520 p., index.

1 La figure de Monge est bien connue des historiens de la Révolution et de l'Empire par les hommages de ses anciens élèves devenus académiciens (Dupin, 1819; Arago, 1853 ; Jomard, 1853) et les études de ses biographes (Louis de Launay, 1933 ; René Taton, 1951 ; Paul Aubry, 1954). Le grand public lui-même a brièvement entendu son nom en 1989, lorsque François Mitterrand, la Mission du bicentenaire et la patrie reconnaissante ont fait entrer le "grand homme » au Panthéon en compagnie de Condorcet et de l'abbé Grégoire, qui ont davantage attiré l'attention. Mais la biographie d'un acteur de la Révolution fait rarement l'objet d'un article dans un grand quotidien national. Or celle de Monge par François Pairault a eu cet insigne honneur, sous la plume de Thierry Lentz (Le Monde, 2 février 2001). Le chapeau soulignait que, "à partir d'archives inédites, François Pairault nous fait redécouvrir le géomètre Gaspard Monge (1746-1818)». En fait, il s'agit bien moins d'une redécouverte que d'une simple découverte pour qui ignore le personnage. De lecture aisée, voire agréable, l'ouvrage pallie sans doute avec bonheur l'absence de biographie disponible en librairie, ce dont le lecteur pourrait se satisfaire. Mais, tout en semblant se conformer aux canons universitaires, il les délaisse, n'apporte rien à la connaissance de l'œuvre, pas grandchose à celle de l'homme, et laisse finalement au lecteur averti un goût un peu amer.

2 On reste en effet confondu de la faiblesse de l'apparat critique, dont la présence effarouche souvent moins les lecteurs que les éditeurs. Certes, les notes biographiques pourront être utiles aux non-spécialistes - malgré quelques regrettables confusions (Hippolyte, fils de Lazare Carnot, devient son frère, p. 22 ; le physicien Pierre Jacotot est 
pris pour son homonyme, le pédagogue Jean-Joseph Jacotot, p. 167) et erreurs (Hachette, le géomètre et disciple à la piété filiale, est faussement envoyé en Égypte, $p$. 144; l'officier aérostier L'Homond, dont Monge a examiné en 1808 un projet de descente aérienne en Angleterre, devient L'Hornond, p.488). Mais jamais elles ne renvoient à de récents travaux, telle l'unique biographie de Hassenfratz par Emmanuel Grison (Presses de l'École des mines, 1996). Et pourtant, aucune bibliographie générale-ni même sommaire- ne vient compenser l'absence quasi-totale de références dans les notes infrapaginales. Comme si l'auteur avait défriché un terrain vierge, comme si rien ou presque n'avait été publié sur rien! Les notes semblent surtout destinées à souligner (sur)abondamment le caractère exceptionnel de l'exploitation du fonds privé sur lequel repose l'ouvrage - plus que le caractère inédit des documents cités, puisque ceux-ci sont souvent déjà connus.

Ainsi, le premier chapitre («Le professeur et le savant [1746-1789]») met en scène l'ancien élève des Oratoriens de Beaune occupant, à dix-huit ans, un emploi modeste à l'École du Génie de Mézières, avant d'y inventer et d'y enseigner la géométrie descriptive, comme successeur (1769) de l'abbé Bossut, professeur de mathématiques, par les soins duquel il entre bientôt à l'Académie royale des sciences et assure un cours au Louvre. Jamais n'apparaissent les travaux sur l'École, initiés par René Taton et Roger Chartier, ni ceux sur l'Académie, nombreux depuis trois décennies dans le sillage de Roger Hahn et de Charles Gillispie (cf. E. BRIAN et C. DEMEULENAERE-DOUYère, dir., Histoire et mémoire de l'Académie des sciences. Guide de recherche, Tec et Doc, 1996). S'il souligne, en 1774, le début d'étroites relations avec le marquis de Castries et avec Pache, l'auteur mentionne à peine l'activité de Monge comme examinateur des gardes de la Marine - et avec elle les études de Dominique Julia et de Michel Vergé-Franceschi sur cette formation - qui explique sa nomination au Conseil exécutif provisoire ( «Un savant ministre de la Marine », du 10 août 1792 au 8 avril 1793). « La défense du territoire » en l'an II ne cite pas même l'ouvrage classique de Camille RICHARD sur Le Comité de Salut public et les fabrications de guerre (1921). Rien de plus pour «Le fondateur de l'École polytechnique ", alors que Janis Langins et Bruno Belhoste ont renouvelé la question autour du bicentenaire, ni sur le professeur de l'École normale de l'an III, malgré l'édition critique des cours de Monge sous la direction de Jean Dhombres (Dunod, 1992). De même, les chapitres « Commissaire des sciences et des arts en Italie (23 mai 1796-26 octobre 1797 » (agrémenté d'une carte pour l'itinéraire de Monge en Italie, p. 191, malheureusement sans repères chronologiques) et «La fondation de la République romaine (26 octobre 1797-26 mai 1798) » soulignent bien l'amitié et l'admiration entre Bonaparte et le mathématicien, mais ils ignorent les travaux récents sur le Trienno en Italie, même ceux de Luigi Pepe sur l'Institut romain, premier Institut national d'une République sœur sur le modèle de celui de Paris, totalement organisé par Monge.

Étonnante exception, dans ce désert historiographique : «L'expédition d'Égypte (26 mai 1798-16 octobre 1799) » est apparemment moins mal traitée à cet égard. Elle a droit à quelques références, parfois même à des ouvrages récents, tels ceux d'Yves LAIssus (L'Égypte, une aventure savante, Fayard, 1998) ou de Nicole et Jean DHOMBrEs (Naissance d'un nouveau pouvoir: sciences et savants en France, 1793-1824, Plon, 1989). Comme ces derniers, mais dix ans après, Pairault ignore toujours l'œuvre de référence de JeanÉdouard GoBy sur l'Institut d'Égypte (Mémoires de l'Académie des inscriptions et belleslettres, 1987) pour retenir " particulièrement » un ouvrage d'Alain PIGEARD qui n'existe pas... - en fait un simple chapitre de compilation, obsolète dès sa parution, dans $L a$ 
campagne d'Égypte de TRANIÉ et CARMIGNIANI (Pygmalion/Watelet, 1988). Quant aux emprunts explicites faits à Christopher Herold (1962) et à Jean-Joël Brégeon (Perrin, 1991), ils ne peuvent faire oublier l'ouvrage de référence de Henry Laurens (Colin, 1989), qui a été réédité en collection de poche en 1998 (Point-Histoire) et a eu le rare privilège d'être traduit en arabe à l'occasion du bicentenaire de l'expédition !

Seuls les derniers chapitres échappent partiellement à cette critique, parce que l'auteur y est davantage sur son terrain et parce que les sujets ont été moins visités par l'historiographie («Le sénateur Monge (1799-1808)», «Les dernières années (1809-1818)»). En définitive, écartant les travaux de ses confrères, l'auteur s'est aussi trouvé prisonnier du piège des archives familiales, au détriment d'une élémentaire diversification des sources, fort abondantes sur Monge. Il décrit sommairement l'importance du fonds relatif à Monge dans les archives du baron de Chaubry, sans préciser qu'une copie partielle - bien connue des historiens - a été déposée à la Bibliothèque de l'Institut de France par Eschassériaux, arrière-petit-fils du savant. Au reste, les «inédits » supposés ne le sont pas toujours, car les archives familiales ont déjà été partiellement exploitées par René TATON (L'œuvre scientifique de Monge, PUF, 1951) et par Paul AUBRY (Monge, le savant ami de Napoléon Bonaparte, Gauthier-Villars, 1954).

6 Monge fut aussi un savant. Les biographies de savants naviguent souvent entre deux écueils : tantôt focalisées sur le scientifique, elles limitent le contexte à quelques anecdotes; tantôt privilégiant surtout l'homme, elles négligent son œuvre scientifique. Pour sa part, Pairault prend soin de se défausser - trop sagement et trop vite (p. 14) sur la « thèse magistrale » (en effet) de René Taton. Sans entrer dans le détail, il aurait pourtant gagné à développer un peu ne fût-ce que la place de la géométrie descriptive inventée par Monge et ses idées sur l'éducation. La compréhension de l'homme luimême et de son charisme auprès de plusieurs générations d'élèves en aurait été mieux assurée, et les prémisses de la fondation de Polytechnique mieux entendues.

Cette nouvelle biographie de Monge est donc plus alléchante par la richesse qu'elle laisse soupçonner encore dans les archives Chaubry, que par des informations nouvelles sur la carrière scientifique et politique de Monge ou sur sa personnalité. Elle constitue une nouvelle couche sédimentaire déjà fossilisée de l'historiographie mongienne plutôt qu'un filon extraordinaire. Pour autant, elle reste une nouveauté bienvenue dans les librairies et pour la constitution d'une bibliothèque révolutionnaire. 